\title{
Survivorship of the Souter-Strathclyde elbow replacement in the young inflammatory arthritis elbow
}

\author{
S. C. Talwalkar, \\ P. K. Givissis, \\ I. A. Trail, \\ D. Nuttall, \\ J. K. Stanley
}

From Wrightington Wigan and Leigh NHS Trust, Wigan, England

S. C. Talwalkar, MCh(Orth), MS(Orth), Upper Limb Fellow

P. K. Givissis, MD, Upper Limb Fellow

I. A. Trail, MD, FRCS, Consultant Orthopaedic Surgeon

D. Nuttall, PhD, Senior Research Fellow

J. K. Stanley, MCh(Orth), FRCS, FRCSE, Professor Hand Research

C/o Mrs Mavis Luya,

Wrightington Hospital, Hall Lane, Wigan WN6 9EP, UK.

Correspondence should be sent to Dr S. C. Talwalkar at 508 Imperial Point, The Quays, Manchester M50 3RA, UK; e-mail: stalwalkar@aol.com

(C)2005 British Editorial Society of Bone and Joint Surgery doi:10.1302/0301-620X.87B7. $15970 \$ 2.00$

$J$ Bone Joint Surg $[\mathrm{Br}]$ 2005;87-B:946-9.

Received 6 September 2004; Accepted 8 October 2004

We divided 309 patients with an inflammatory arthritis who had undergone primary elbow replacement using the Souter-Strathclyde implant into two groups according to their age. The mean follow-up in the older group (mean age 64 years) was 7.3 years while in the younger patients (mean age $\mathbf{4 2}$ years) it was 12 years. Survivorship for three different failure end-points (revision, revision because of aseptic loosening of the humeral component, and gross loosening of the humeral implant), was compared in both groups.

Our findings showed that there was no significant difference in the incidence of loosening when young rheumatoid patients were compared with an older age group.

Over the last few decades there has been an improvement in the results of total elbow arthroplasty. This may be because of superior prosthetic design or technical changes such as better cementation. However, these procedures tend to be reserved for older patients with a lower demand because of the perceived risk of early loosening in young active patients.

Several studies on the Souter-Strathclyde elbow replacement (Stryker Howmedica Osteonics, Allendale, New Jersey) have reported predictable relief from pain, good to excellent function and a survivorship of between $77.4 \%$ and $85 \%$ at ten years. ${ }^{1,2}$ There are few reports on the use of this prosthesis specifically in a younger age group. Our aim, therefore, was to compare the survivorship of the Souter-Strathclyde elbow replacement in patients under 50 years of age at surgery with that in older patients.

\section{Patients and Methods}

From a database of 309 patients in whom Souter-Strathclyde elbow replacements had been performed at our institution between 1984 and 2000 there were 36 who were younger than 50 years of age at the time of surgery. Ten had undergone bilateral surgery. Consequently, 46 elbow replacements were included. Radiological data on the remaining 263 elbow replacements were used in a survivorship analysis. Table I gives details of the two groups.

In the younger than 50 years of age group there were five men and 31 women with a mean age of 42.2 years (27 to 49 ) at the time of surgery. Those over 50 years of age (mean age 64.5 years (50 to 82 ); gender distribution male:female, 1:3) had a shorter follow-up of 7.3 years ( 3 to 16 ) compared with 12 years ( 5 to 16 ) in the group under 50 years of age.

The indication for surgery in all patients was painful inflammatory joint disease. Those aged under 50 years had seropositive arthritis. In the older group, four patients had psoriatic arthritis and the remainder had rheumatoid arthritis. In all patients there was considerable destruction of the elbow (Larsen grade 3, 4 and 5). ${ }^{3}$

Table I. Details of the two groups $<50$ years of age and $>50$ years of age

\begin{tabular}{llc}
\hline & $<50$ years & $>\mathbf{5 0}$ years \\
\hline Number of operations & 46 & 263 \\
Mean age in yrs (SD; range) & $42.2(6.3 ; \mathbf{2 7}$ to 49$)$ & $64.5(7.4 ; 50$ to 82) \\
Mean follow-up in yrs (range) & $12 \quad(5$ to 16$)$ & 7.3 (3 to 16) \\
Standard humeral implant & 12 & 168 \\
Long-stemmed humeral implant & 34 & 95 \\
Larsen grade & & \\
$\quad 3$ & 0 & 2 \\
$\quad 4$ & 25 & 140 \\
$\quad 5$ & 21 & 121 \\
Length of study in years & 16 & 16 \\
\hline
\end{tabular}




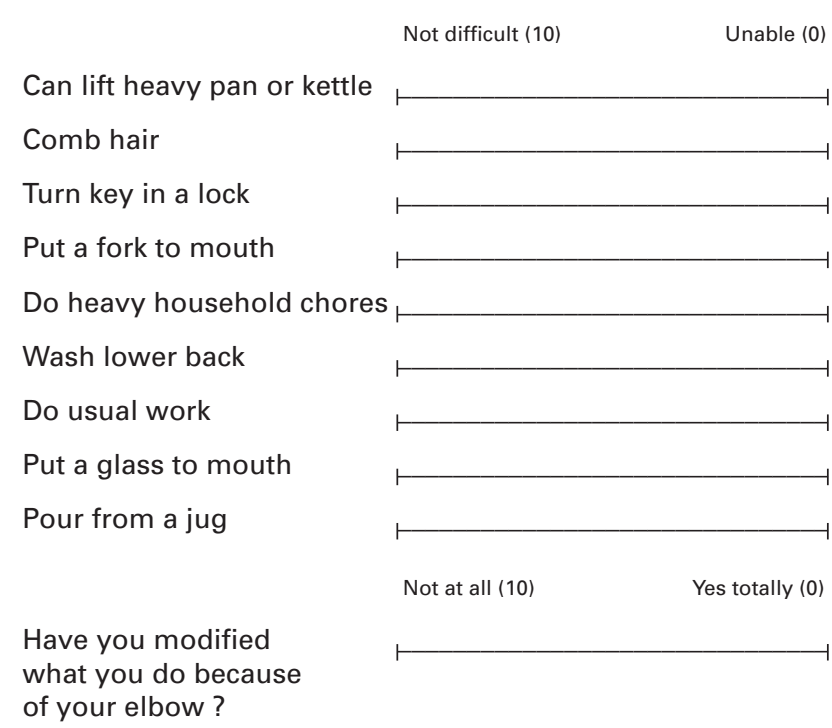

Fig. 1

Diagram showing the functional score questionnaire (maximum score $=$ 100 points).

Clinical evaluation. We reviewed the case notes, operative records and radiographs. All patients were examined by one of the researchers.

Pain was recorded using a visual analogue scale (VAS) from 0 (no pain) to 10 (maximum pain). This was assessed at rest, at night and at its worst. The pre-operative range of movement (flexion, extension, pronation/supination) was compared with that at the most recent assessment. All patients were asked to complete a questionnaire to evaluate their functional status. This included a series of ten questions based upon their activities of daily living (Fig. 1). The questions were graded on a VAS of 0 to 10 giving a maximum possible score of 100 . Finally, patients were also asked for a subjective assessment of their elbow replacement (excellent, good, satisfactory, no change, poor).

Radiological analysis. We used the Wrightington method ${ }^{4}$ for numbering and measuring migration and demarcation on radiographs (Fig. 2). Standardised anteroposterior and lateral radiographs of the elbow were taken after surgery and at assessment. We obtained 21 variables of demarcation zone from each radiograph, reducing them by principal-component analysis to just two indices, $\mathrm{H}$ and $\mathrm{U}$, which represented loosening of the humeral and ulnar components, respectively. ${ }^{5}$

Statistical analysis. Survival tables and curves were calculated for three different end-points, namely revision for any reason, revision because of aseptic loosening (Hloose revisions) and gross loosening of the $\mathrm{H}$ implant (Hindex $>1$ ) and are presented graphically with Kaplan-Meier plots (Figs 3 to 5). The log-rank test was used to compare the survival between the two groups. Analyses were conducted using SPSS 11 (SPSS Inc, Chicago, Illinois). All reported $\mathrm{p}$ values were two-tailed.

\section{Results}

Of the 36 patients included in the group under 50 years of age, 26 (31 elbow replacements) were available for clinical assessment. One patient had recently died because of reasons unrelated to the surgery. In five patients there were no pre-operative clinical data regarding the range of movement and pain scores. Four patients did not attend the research clinic. However, all 46 elbow replacements had pre-operative and recent radiographs.

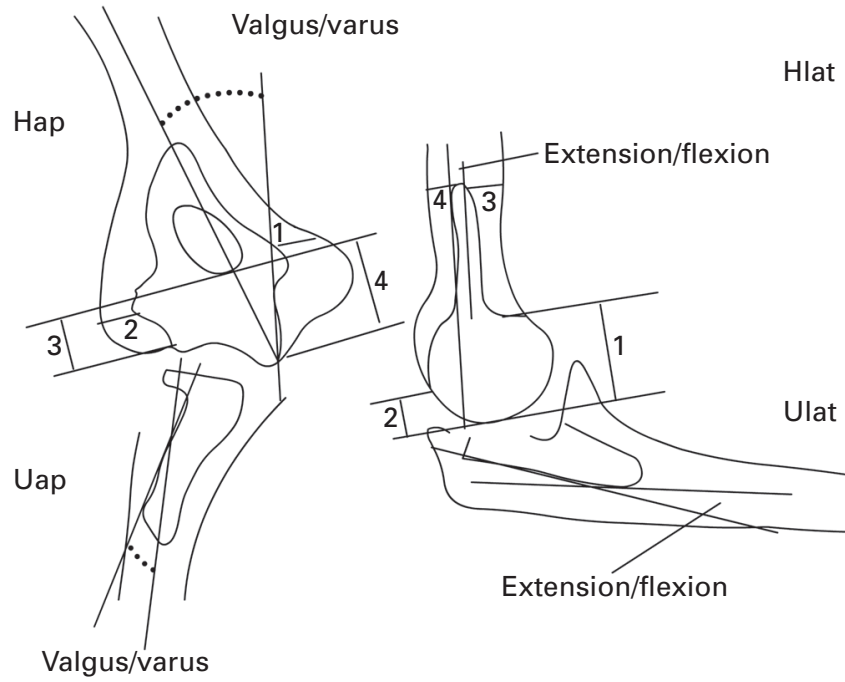

Fig. $2 a$
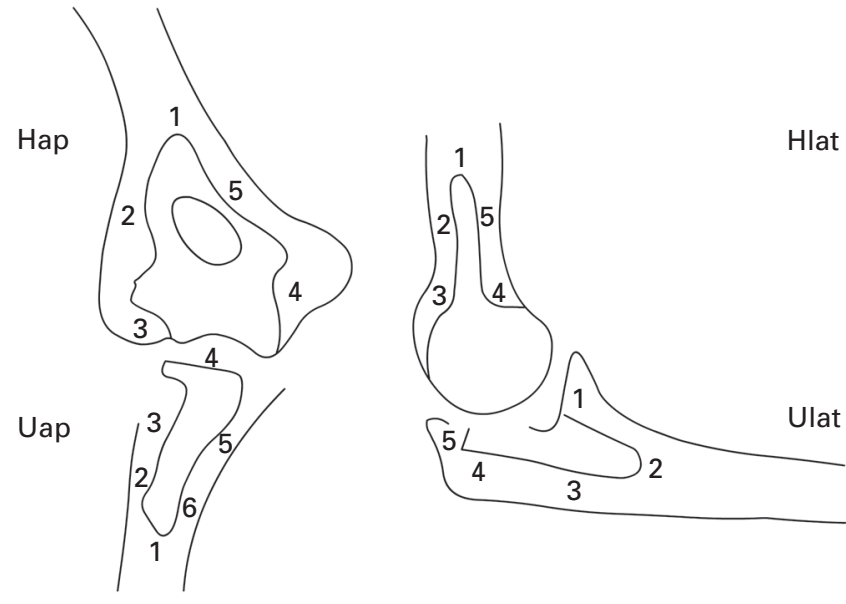

Fig. $2 b$

Diagrams showing a) the angle and measurement taken from each radiograph and b) the areas of radiological demarcation around the elbow arthroplasty. 


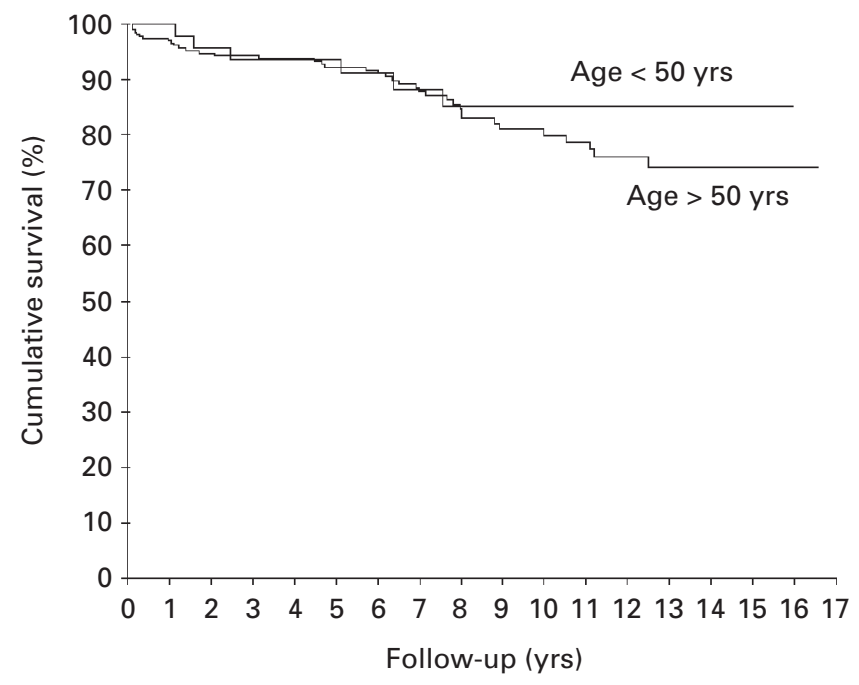

Fig. 3

Kaplan-Meier survival curve for revision for any reason.

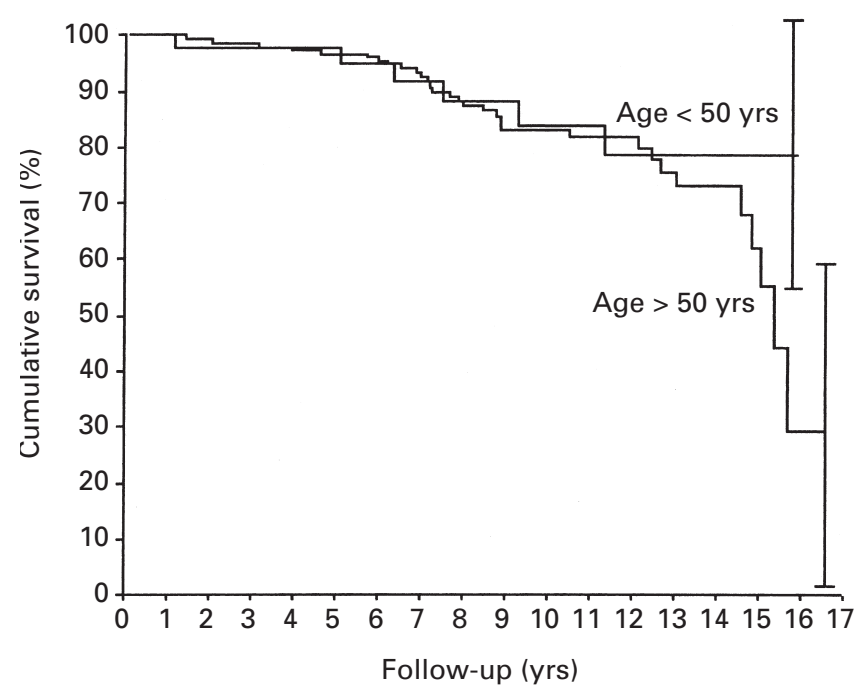

Fig. 5

Kaplan-Meier survival curve for gross loosening of the $\mathrm{H}$ implant (Hindex $>1)$. The wide confidence intervals indicate low sample numbers.

Relief from pain. Assessment of pain showed an improvement in the pain score using the VAS from a mean of seven (6 to 10$)$ in the pre-operative period to two $(0$ to 7$)$ at assessment in clinic. Twenty-four replacements were completely free from pain (VAS 0 or 1). Five elbow replacements had severe pain (VAS score 6) while the remaining two elbows had moderate pain (VAS 2 to 5). No patient had pain at rest.

Range of movement. Before surgery the mean arc of movement was $49^{\circ}\left(5^{\circ}\right.$ to $\left.80^{\circ}\right)$. Post-operatively it was $139^{\circ}\left(90^{\circ}\right.$

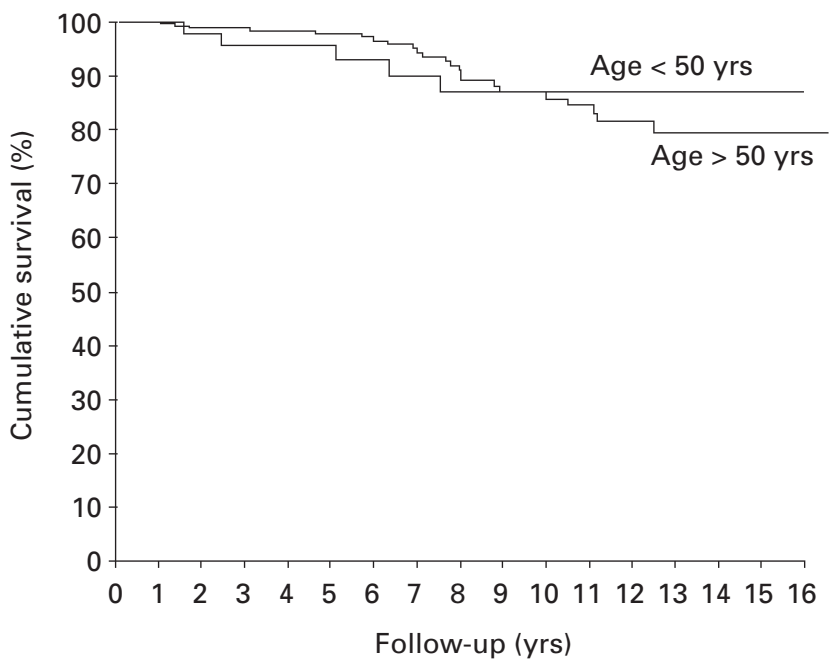

Fig. 4

Kaplan-Meier survival curve for revision because of aseptic loosening (Hloose revisions).

to $\left.140^{\circ}\right)$. There was a gain in flexion in 29 elbows, a loss in one and no change in one. The mean gain in flexion was $90^{\circ}$. Forearm rotation after surgery was increased from a mean of $29^{\circ}\left(0^{\circ}\right.$ to $\left.180^{\circ}\right)$ to $102^{\circ}\left(55^{\circ}\right.$ to $\left.180^{\circ}\right)$.

Functional evaluation. Subjectively, 23 of 26 patients were satisfied with their surgery (excellent to good), one had a poor result and two patients had not changed from their pre-operative state. The mean functional score at follow-up using our scoring system was 70 of a maximum of 100 . This compared with the pre-operative mean of 46 .

Complications. Six patients developed aseptic loosening and required their prosthesis revised. All had the short-stem prosthesis. Five required revision of the humeral and one revision of the ulnar component.

Symptoms of ulnar neuropathy developed in three patients in the immediate post-operative period. One developed weakness in the distribution of the ulnar nerve while two had transient paraesthesiae along the medial two fingers which improved over a period of three months. At clinical review the patient with motor loss still had weakness of the interossei but the sensory neuropathy had recovered completely.

There were three superficial infections which were successfully treated by an extended course of intravenous antibiotics. One patient had a large myositic mass and a poor result with severe pain and a stiff elbow.

There was one fracture of the lateral column which was detected on the post-operative radiographs but remained largely asymptomatic and had united at the patient's last assessment.

Survivorship analysis. When all causes were considered the risk of having a revision was analysed. The cumulative survival for patients aged $>50$ years was $74 \%$ after 16 years, 
while the same cumulative survival for those aged $<50$ years was higher $(85 \%)$, although this was not statistically significant (log-rank test, $\mathrm{p}=0.3994$ ) (Fig. 3).

When revision because of aseptic loosening (Hloose revision) was considered, the cumulative survival for patients aged $>50$ years was $80 \%$ after 16 years but $87 \%$ for those aged $<50$ years. This difference was not statistically significant, as shown in Figure 4 (log-rank test, $\mathrm{p}=0.8193$ ).

The risk of having gross loosening of the $\mathrm{H}$ implant (Hindex $>1$ ) for both age groups is shown in Figure 5. The rate of Hindex > 1 after 16 years in the older patients was $29 \%$ while in the younger patients it was $79 \%$; this difference was not significant (log-rank test, $p=0.3467$ ), explained by the widening confidence intervals as the number of patients considered at 16 years is significantly reduced (Fig. 5).

\section{Discussion}

Despite improvements in medical treatment for rheumatoid arthritis, progressive joint destruction leading to severe disability may occur in some young patients. Temporary measures, including the use of splints and soft supports, or other modalities such as cold or heat, may be helpful when the pain is not severe and dysfunction not limiting. When medical therapy fails to control symptoms these patients may require surgical intervention. Total elbow arthroplasty is a tempting alternative for the young adult as relief from pain is likely and functional improvement is predictably good or excellent. ${ }^{6}$ However, there are potential long-term disadvantages with loosening and loss of bone. Loosening may be associated with return of pain and loss of function. The technical challenge of revision surgery can be considerable and the outcome of surgery less predictable. Consequently, most investigators have, in the past, recommended total elbow arthroplasty for patients over the age of 60 years. $^{7,8}$

The series reported by Connor and Morrey, ${ }^{9}$ which included patients with juvenile rheumatoid arthritis compares favourably in many respects with our study. They reported excellent results in $52 \%$ of 23 elbow replacements which had been followed up for at least two years. There was a marked improvement in the pain score, with 22 of the 23 elbows having little or no pain. However, the improvement in the range of movement was not as marked as has been reported in the literature. Despite this, functional ability was consistently enhanced with $78 \%$ of patients reporting no difficulties with activities of daily living.

Our findings differed from those of Connor and Morrey ${ }^{9}$ in some respects. We found that total elbow arthroplasty provided relief from pain and excellent patient satisfaction with a marked improvement in the range of movement. The reason for the lack of improvement in range of movement in the former study was attributed to the severe osseous atrophy, deformity and recalcitrant contractures which occur in juvenile rheumatoid arthritis. When there was loosening of the humeral component the tip moved anteriorly onto the anterior humeral cortex. Previous studies by Trail et $\mathrm{al}^{4,5}$ have demonstrated that this loosening can be predicted by the rate of change in the angle of extension of the prosthesis. All six replacements which loosened were also short-stemmed humeral prostheses. Previously published work from this institution ${ }^{4}$ which compared shortand long-stemmed Souter-Strathclyde prostheses, has shown a significant rate of loosening and revision in the short-stemmed version of this elbow replacement. Hence the long stem is now preferred.

Our study showed that when age is considered as a factor in comparing long-term survivorship there does not appear to be any difference between patients less than 50 years of age and those over 50 years of age at surgery for all three end-points. In the third category, when the risk of having gross loosening of the $\mathrm{H}$ implant (Hindex > 1) for both age groups is considered, those $>50$ years of age had a probability of survival of $29 \%$ at 16 years compared with $79 \%$ in those under 50 years of age. This apparent large difference is misleading because the calculation depends upon the number of subjects under investigation at any given time. The distribution of the number of terminal events at distinct times for the two survival curves was compared and the log-rank statistic indicated that this difference was insignificant.

We wish to thank Mrs. Diane Allmark and Mrs. Stella Crank for their administrative help during the project.

Although none of the authors has received or will receive benefits for personal or professional use from a commercial party related directly or indirectly to the subject of this article, benefits have been or will be received but will be directed solely to a research fund, foundation, educational institution, or other nonprofit organisation with which one or more of the authors are associated.

\section{References}

1. Ikavalko M, Lehto MU, Repo A, Kautiainen H, Hamalainen M. The SouterStrathclyde elbow arthroplasty: a clinical and radiological study of 525 consecutive cases. J Bone Joint Surg [Br] 2002;84-B:77-82.

2. Van der Lugt JC, Geskus RB, Rozing PM. Primary Souter-Strathclyde total elbow prosthesis in rheumatoid arthritis. J Bone Joint Surg [Am]2004;86-A:465-73.

3. Larsen A. A radiological method for grading the severity of rheumatoid arthritis. Scan J Rheumatol 1975;4:225-33

4. Trail IA, Nuttall D, Stanley JK. Comparison of survivorship between standard and long-stem Souter-Strathclyde total elbow arthroplasty. J Shoulder Elbow Surg 2002; 11:373-6.

5. Trail IA, Nuttall D, Stanley JK. Survivorship and radiological analysis of the standard Souter-Strathclyde total elbow arthroplasty. J Bone Joint Surg [Br] 1999;81-B: $80-4$

6. McAuliffe JA. Surgical alternatives for elbow arthritis in the young adult. Hand Clin 2002;18:99-111.

7. Mansat $\mathbf{P}$, Morrey BF. Semi-constrained total elbow arthroplasty for ankylosed and stiff elbows. J Bone Joint Surg [Am] 2000;82-A:1260-8.

8. Morrey BF, Adams RA, Bryan RS. Total elbow replacement for post-traumatic arthritis of the elbow. J Bone Joint Surg [Br] 1991;73-B:607-12.

9. Connor PM, Morrey BF. Total elbow arthroplasty in patients who have juvenile rheumatoid arthritis. J Bone Joint Surg [Am]1998;80-A:678-88. 